Reprod. Nutr. Dévelop. 1980, 20 (2), 457-468.

\title{
Effet de la présence du mâle sur les mécanismes de reproduction chez la femelle des mammifères.
}

\author{
par J.P. SIGNORET
}

Station de Physiologie de la Reproduction I.N.R.A., Nouzilly 37380 Monnaie, France.

Summary. Effect of the male presence on the reproductive mechanisms in female mammals.

In various physiological situations, the presence of the male may interfere with the timing of the physiological events of female reproduction. Contact with the male hastens puberty in a variety of species, provided that the female has reached a given age and weight.

The male presence may induce synchronized ostrus in the adult female. In the case of seasonal or lactation anoestrus in ewes, contact with the ram increases the pulsatile release of $\mathrm{LH}$ within a few minutes, resulting in a preovulatory surge $24 \mathrm{hrs}$ later, followed by ovulation, but usually no behavioural œstrus. However, the development of the corpus luteum is abnormal in half of the cases, leading to an abnormally short 7-day cestrous cycle.

During cestrus itself, the male presence reduces markedly the duration of sexual receptivity and hastens ovulation.

Direct contact is not needed to produce these effects which can be mediated by the sense of smell and also by other senses, acting in a cumulative, and possible substifutive, way.

Male-induced ovulation would ensure a higher probability of fertilization in species in which the males and females are not permanently associated. Furthermore, the resulting synchronized reproduction might have an adaptative value by reducing predation at birth in wild species. Such effects could also be used for the control of domestic animal production.

L'existence d'un fonctionnement cyclique régulier de l'ovaire est une caractéristique commune à de nombreuses femelles de mammifères (rongeurs, ongulés, primates). Les mécanismes du cycle œstrien et de l'ovulation ont été très largement élucidés ; chez les espèces où le fonctionnement ovarien cyclique est limité à certaines périodes de l'année, le rôle déterminant de la durée journalière d'éclairement a été mis en évidence et ses modalités d'action sont également bien étudiées.

Dans ces domaines, l'importance des connaissances acquises peut se mesurer par la précision et la multiplicité des interventions qui sont l'application pratique - tant dans l'espèce humaine (traitement de la stérilité ou contraception) que pour la maîtrise de la fertilité des espèces domestiques. 
Cependant, les mécanismes mis en évidence ne suffisent pas à rendre compte de tous les aspects du déroulement des phénomènes de la reproduction tels qu'ils peuvent se produire dans les conditions naturelles ou dans la pratique de l'élevage. En effet, on peut observer notamment des anomalies par rapport à ce que devrait être la probabilité statistique d'apparition de l'activité sexuelle. Ni les variations des paramètres physiques, ni l'influence des disponibilités alimentaires ne peuvent rendre compte de tels phénomènes qui semblent résulter d'interactions comportementales, en particulier liées à la présence du mâle. De tels phénomènes sont bien connus chez les oiseaux : la présence du partenaire sexuel, les émissions sonores spécifiques, la présence d'un nid, sont autant de facteurs dont le rôle a pu être mis en évidence. Ils sont bien moins classiques chez les mammifères.

Nous nous proposons de passer en revue les différents cas où des phénomènes de ce genre ont pu être mis en évidence en envisageant dans chaque situation les voles sensorielles et les mécanismes impliqués.

La présence du mâle peut agir sur la physiologie sexuelle de la femelle dans diverses sifuations :

- lors de l'apparition de la puberté,

- chez l'adulte pour modifier le déroulement du cycle œstrien,

- lors de l'œstrus sur les mécanismes de l'ovulation,

- après la fécondation sur les mécanismes de mise en place du corps jaune de gestation. Ce dernier cas, où la présence d'un mâle étranger perturbe l'implantation des œufs fécondés par un premier accouplement - effet Bruce - semble limité à une seule espèce, la souris. Malgré son intérêt, il ne s'agit que d'un cas très particulier que nous n'envisagerons pas dans cette revue.

\section{Apparition de la puberté}

1. Le phénomène. - L'apparition de la puberté chez les jeunes femelles des espèces domestiques donne parfois lieu à des phénomènes de synchronisation qui sont mis à profit par les éleveurs pour l'organisation de la reproduction. C'est ainsi que le transport ef la mise en présence du mâle provoquent une apparition du premier œstrus de 4 à 7 jours plus tard chez de jeunes truies (du Mesnil du Buisson et Signoret, 1962 ; Brooks et Cole, 1970 ; Paterson, 1978), l'agnelle (Dyrmundsson et Lees, 1972) ou la souris (Vandenbergh, 1967) (tabl. 1). Ce phénomène est particulièrement net pour une souche donnée, lorsque les animaux atteignent un âge et un poids précis ; avant, le taux de réponse est faible, plus tard, les femelles semblent avoir spontanément atteint la puberté. Il pourrait ainsi exister un état instable de « prépuberté » où la présence du mâle jouerait un rôle déclencheur. Chez la truie, par exemple, 23 p. 100 des jeunes femelles abattues à un âge où elles répondent à la présentation du mâle, sont dans un stade de développement utérin et ovarien qui évoque l'imminence de la puberté (du Mesnil du Buisson et Signoret, 1962). On imagine que de tels animaux sont dans les conditions où ils puissent avoir une capaciłé de réponse rapide.

2. Voies sensorielles. - Cette puberté précoce ne nécessite pas le contaci avec le mâle. Elle peut être obtenue, chez la Souris, en exposant de jeunes femelles à l'odeur 
de la litière d'un mâle adulte ou à son urine (Vanderbergh, 1969 ; Kennedy et Brown, 1970 ; Vanderbergh, Drickamer et Colby, 1972 ; Colby et Vandenbergh, 1974). La présence d'un composé actif dans l'urine est sous la dépendance des androgènes. II s'agirait d'un élément protéique présent dans l'urine du mâle intact, mais, les efforłs pour l'identification de cette substance n'ont pas, pour l'instant, abouti (Vandenbergh, Whitsett ef Lombardi, 1975).

\section{TABLEAU 1}

Exisfence d'un effet mâle sur l'apparition de la puberté

\begin{tabular}{|c|c|c|}
\hline Auteur & Espèce & Phénomène observé \\
\hline Brooks et Cole, 1970 & Porcins & $\begin{array}{l}11 / 12 \text { jeunes truies présentées au mâle à l'âge de } 165 \text { jours } \\
\text { viennent en œstrus dans les } 10 \text { jours. Chez les témoins, } \\
\text { la première da venir en œstrus le faił à } 190 \text { jours. A } \\
215 \text { jours, } 7 / 12 \text { sont pubères. }\end{array}$ \\
\hline $\begin{array}{l}\text { Du Mesnil du Buisson ef } \\
\text { Signoret, } 1962\end{array}$ & Porcins & $\begin{array}{l}277 / 1043 \text { jeunes truies sont en œestrus } 4-6 \text { jours après lo } \\
\text { présentation au mâle. Ce taux passe de } 20 \text { à } 35 \mathrm{p} .100 \\
\text { des femelles dont le poids passe de } 80 \text { d } 100 \mathrm{~kg} \text {. }\end{array}$ \\
\hline $\begin{array}{l}\text { Dyrmundsson et Lees, } \\
1972\end{array}$ & Ovins & $\begin{array}{l}\text { Sur } 25 \text { agnelles mises en présence du bélier à l'âge de } \\
145 \text { jours on n'observe aucun groupage d'œestrus. } \\
\text { Sur un lot de } 25 \text { présentées au bélier à } 195 \text { jours, } 13 \text { sont } \\
\text { en œstrus } 18-22 \text { jours après. }\end{array}$ \\
\hline Vandenbergh, 1967 & Souris & $\begin{array}{l}\text { Pour des souris mises en présence du mâle à l'âge de } \\
21 \text { jours, l'ouverfure vaginale est observée en moyenne } \\
\text { à } 30,5 \text { jours et le } 1 \mathrm{er} \text { cestrus d̀ } 37,1 \text { jours, contre } 34,9 \\
\text { ef } 57,1 \text { respectivement pour les témoins isolés. }\end{array}$ \\
\hline
\end{tabular}

Cependant la voie sensorielle olfactive n'est pas seule en cause. La présence d'un mâle castré qui, seul, est sans action, accroît l'efficacité de l'exposition à l'urine de mâle (Bronson et Maruniak, 1975) le contact avec une femelle androgénisée à la naissance est également efficace (Drickamer, 1974).

Toutefois, on ne peut exclure, dans certains cas, l'effet d'un phénomène de stress qui pourrait résulter d'un changement dans les conditions d'élevage : présence d'animaux inconnus, poursuites sexuelles, etc... Un tel phénomène existe chez la brebis (Braden et Moule, 1964) et chez la truie (Signoref, 1970). Cependant, chez les porcins, Bourn ef al., 1975 onf pu séparer les deux phénomènes et montrer l'existence indépendante d'un effet mâle.

3. Le mécanisme de l'accélération de la puberté par la mise en présence du mâle a été étudié chez la souris par Bronson et Desjardin (1974). Une augmentation du taux de LH circulant est observée dès $1 \mathrm{~h}$ après la mise en présence du mâle. Ensuite on observe un accroissement considérable du taux d'œstradiol qui, en $12 \mathrm{~h}$, est multiplié par un facteur 15-20.

Le poids de l'utérus s'accroît rapidement et, le 3 e jour, œstrogènes, $\mathrm{FSH}$ et LH présentent une évolution analogue à celle observée chez l'adulte et qui aboutit à une ovulation accompagnée d'œstrus. 


\section{Modifications du cycle œstrien chez l'adulte}

1. Le phénomène. - Lorsque des femelles adultes sont mises en présence du mâle, la répartition des œstrus observés est parfois très différente de ce qui devrait résulter d'une probabilité statistique : c'est ainsi que chez la souris, Whiften (1956) observe que 46 p. 100 des souris adultes s'accouplent le $3^{\mathrm{e}}$ jour après leur présentation au mâle, alors qu'une répartition régulière devrait être attendue pendant les 4 ou 5 premiers jours, ce qui correspond au cycle œstrien normal. De même, chez la brebis, en début de saison sexuelle, 80 p. 100 environ s'accouplent entre le $15^{\mathrm{e}}$ et le $24^{\mathrm{e}}$ jour après l'introduction du bélier (Prud'hon et Denoy, 1969). Ce phénomène signalé pour la première fois par Underwood, Shier et Davenport (1944) a été confirmé par de nombreux auteurs, il est également mentionné chez la chèvre (Shelton, 1960).

Il est établi que ce phénomène résulte, dans certains cas, d'une modification de la durée du cycle œstrien. Il en est ainsi chez la souris de laboratoire (Whitten, 1958) ou la souris sauvage (Chipman et Fox, 1966), le péromyscus (Marsden et Bronson, 1964), le rat (Hughes, 1964 ; Aron, Roos et Asch, 1968 ; Aron et Chateau, 1969). Cette dernière équipe a montré que la présence du mâle pouvait raccourcir à 4 jours la durée du cycle de femelles ayant normalement un infervalle de 5 jours entre les cestrus.

Le groupage des cestrus peut aussi être dô à la rupture d'un état de repos sexuel. C'est ainsi que chez la souris, des femelles groupées en l'absence du mâle présentent de fréquentes irrégularités de cycle œstrien, pouvant aboutir à un anœstrus temporaire (Van der Lee et Boot, 1955 ; Whitten, 1959). La présence du mâle induit une reprise synchronisée des cycles. Il en est de même chez la brebis et la chèvre : l'effet du mâle apparaît à la fin de la période saisonnière d'ancstrus. Toutefois, il peut être mis en évidence dans d'autres cas : lors de la fin de la période saisonnière d'activité ovarienne, l'isolement temporaire suivi de la réunion avec le mâle prolonge l'existence de cycles œstriens et retarde l'entrée en anœstrus de la brebis (Lishman et Hunter, 1966).

L'allaifement est une autre cause de suspension des cycles œstriens. La durée de l'anœstrus de lactation est réduite par la mise en présence du mâle chez la brebis (Mauléon ef Dauzier, 1965), la vache (Signoret, non publié), la truie (Rowlinson et Bryant, 1974).

Enfin, la sous-alimentation peut également perturber les cycles œestriens. Or, la présence d'un mâle réduit la fréquence de cycles anormaux chez la rate (Cooper ef Haynes, 1967).

2. Voies sensorielles. - L'importance du canal sensoriel olfactif est mis en évidence dans de nombreux travaux : chez la souris, Whitten (1956) montre que le groupage des accouplements ne nécessite pas le contact non plus que l'ouïe (Whitten, 1966) ou la vue (Lamond, 1959). La litière souillée par le mâle est efficace (Whitten, 1958) ou son urine (Marsden et Bronson, 1964), il en est de même chez le rat (Aron ef Chateau, 1971). Plusieurs auteurs ont émis l'hypothèse de la sécrétion d'une phéromone par les glandes préputiales (Albrecht, 1967 ; Chipman et Albrecht, 1974), mais l'urine prélevée directement dans la vessie reste efficace (Bronson et Whitten, 1968). Ces mêmes auteurs montrent en outre que la substance active peut être produite sous l'influence des androgènes par les castrats des deux sexes. 

1960).

Chez la brebis, ni le confact ni la vue ne sont nécessaires (Watson et Radford,

L'ensemble de ces données incite donc à attribuer à l'odorat le rôle déterminant dans ce phénomène. Cependant, chez les ovins, une série d'observations montre que le mécanisme de l'effet mâle est, au moins dans cette espèce, beaucoup plus complexe. En effet, pour que la présence du mâle soit efficace, elle doit être un fait nouveau : le groupage des œstrus n'apparaît pas en début de saison sexuelle, si le bélier est en permanence associé aux brebis, mais seulement lorsqu'il les rejoint, après en avoir été éloigné temporairement (Riches et Watson, 1954). Il en esí de même en fin de saison sexuelle : seul un isolement temporaire, suivi d'une réunion avec le mâle, permet la prolongation des cycles œstriens, que l'on n'observe pas lorsque des béliers vasectomisés sont présents en permanence (Lishman et Hunter, 1966).

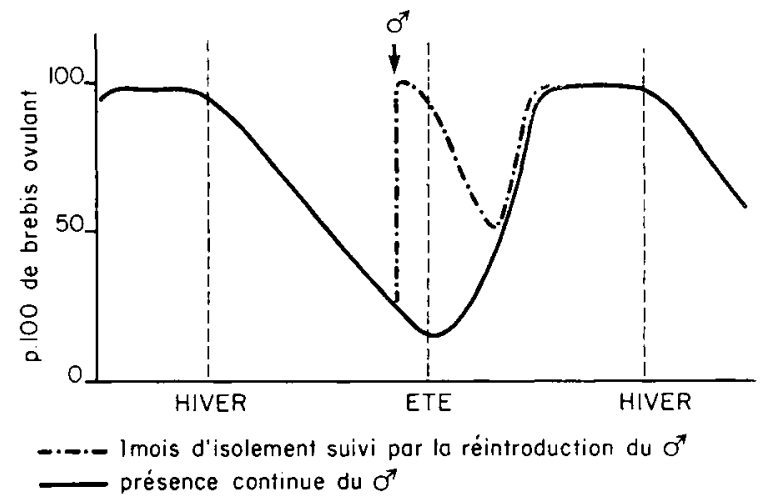

FIG. 1. - Evolution du pourcentage de brebis Merinos présentant des ovulations au cours de l'année (Oldham, 1980).

Oldham (1980) a montré que, chez la brebis Merinos, il existait une période d'ancestrus aussi marquée si les femelles sont isolées que si elles sont en permanence avec des mâles vasectomisées, par contre, à n'ímporte quel moment, le bélier peut déclencher l'apparition de l'œstrus. En l'absence de fécondation, l'anœstrus s'établit à nouveau (fig.1) et il faut environ un mois de séparation pour qu'un nouvel effet mâle redevienne possible. Un phénomène analogue est signalé chez l'alpaca (FernandezBaca, Sumar et Novoa, 1972).

3. Mécanisme. - Chez les ovins, les mécanismes qui interviennent lors de la rupture de l'anœstrus sous l'influence du mâle, ont été étudiés en détail.

Les différents cas d'effet mâle signalés semblaient recouvrir des phénomènes relativement lents dont le déroulement demandait plusieurs jours. Or, l'examen systématique des ovaires par endoscopie a permis à Oldham, Martin et Knight (1979) de mettre en évidence l'existence d'un phénomène très rapide : la quasi-totalité des brebis présentées au mâle ovulent environ $48 \mathrm{~h}$ plus tard (fig. 2). Cette première ovulation n'est pas, le plus souvent, accompagnée d'œstrus et est suivie dans la moitié des cas par un cycle court, 6-7 jours, conséquence d'un développement anormal du corps jaune (Oldham et Martin, 1979). La rapidité de cette réponse suppose une réaction très rapide 


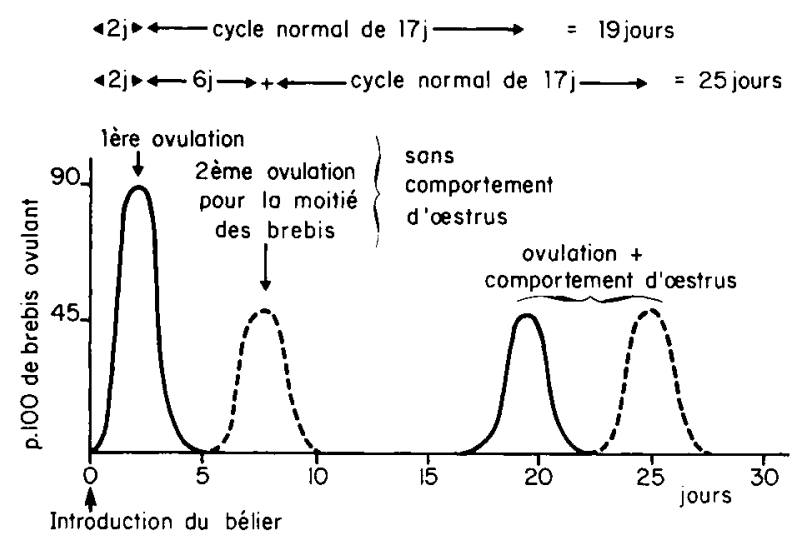

FIG. 2. - Effet de l'introduction du bélier en période d'anœestrus sur l'ovulation, le cycle œstrien ef le comportement sexuel de brebis Merinos (Oldham et Martin, 1979).

des mécanismes contrôlant la sécrétion des hormones hypophysaires gonadotropes. Chesworth et Tait (1974) ont signalé dans les $10 \mathrm{~h}$ suivant l'introduction du bélier une légère augmentation moyenne du taux de $\mathrm{LH}$ circulant, tandis que Knight, Peterson et Payne (1978), Oldham, Martin et Knight (1979), Cognié Poindron et Orgeur (1978) ont mis en évidence l'existence d'un pic ovulatoire environ $36 \mathrm{~h}$ après la présentation au mâle (łabl. 2).

\section{TABLEAU 2}

Données bibliographiques concernant l'intervalle entre la présentation des brebis au bélier ef l'apparition de la décharge ovulante de $\mathrm{LH}$

\begin{tabular}{|c|c|c|}
\hline Auteur & Race & $\begin{array}{c}\text { Intervalle } \\
\text { (h) }\end{array}$ \\
\hline $\begin{array}{l}\text { Knight ef al., } 1978 \ldots \ldots \ldots \\
\text { Oldham ef al., } 1979 \ldots \ldots \ldots \\
\text { Cognié ef al., } 1978 \ldots \ldots\end{array}$ & $\begin{array}{l}\text { Romney } \\
\text { Merino } \\
\text { Préalpes } \\
\text { Ile-de-France }\end{array}$ & $\begin{array}{l}36 \\
27 \\
25 \\
20\end{array}$ \\
\hline
\end{tabular}

La dynamique complète du phénomène a été mise en évidence par Poindron ef al. (1979). Aussi bien lors de l'anœstrus saisonnier que pendant la lactation, l'introduction du bélier provoque, dans les minutes qui suivent, une accélération de la sécrétion pulsatile de LH dont le niveau moyen s'accroît el qui évolue vers l'apparition d'un pic préovulatoire 24 à 36 h plus tard (fig. 3).

Il apparaît donc que des signaux spécifiques émis par le mâle peuvent déclencher très rapidement le fonctionnement hypophysaire qui aboutit à l'ovulation.

\section{Lors de l'cestrus et de l'ovulation}

La notion d'ovulation spontanée, opposée à celle d'ovulation provoquée, implique le jeu de sécrétions hormonales excluant les stimulations sensorielles. Toutefois, la présence du mâle et l'activité sexuelle elle-même peuvent en modifier le déroulement temporel. 

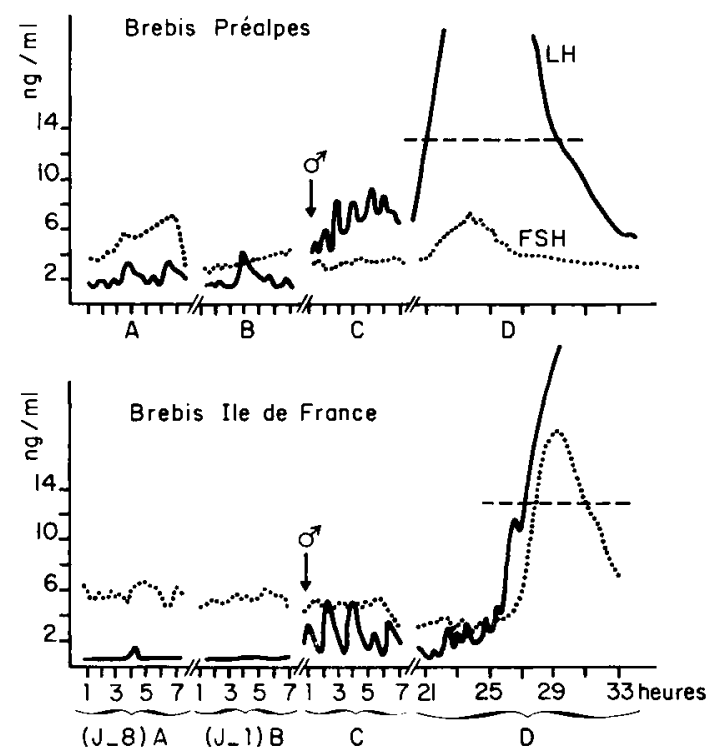

FIG. 3. - a) Effet de la présentation au bélier sur l'évolution de la sécrétion de LH ef FSH chez une brebis Préalpes et une brebis lle-de-France en anœstrus saisonnier. Les prélèvements de sang ont été effectués à un intervalle de $20 \mathrm{mn}$ pendant $6 \mathrm{~h}$, à deux reprises avant l'introduction du bélier (A et B) ef à la suite de celle-ci (C) enfin de 21 à 33 h après (D) (Cognié, Poindron et Orgeur, 1978).

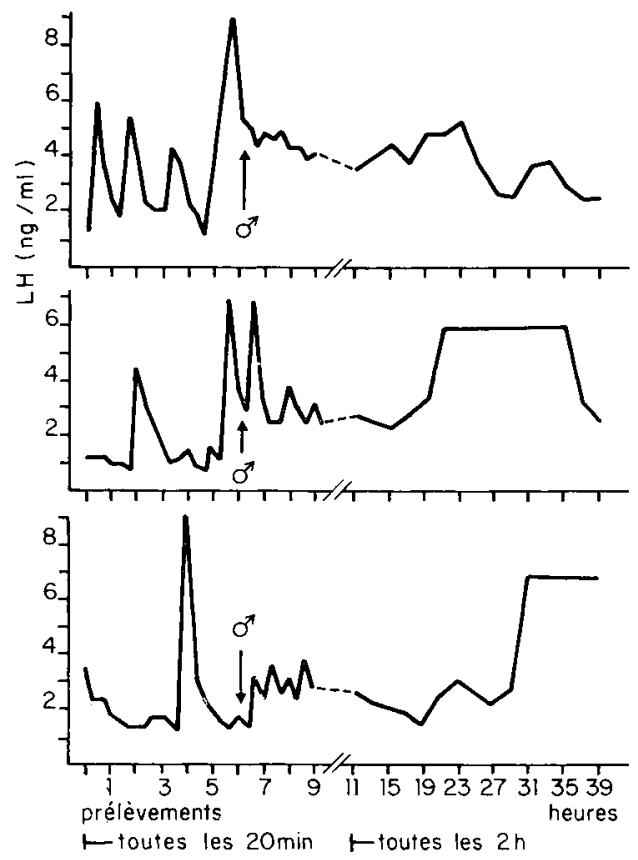

FIG. 3. - b) Evolution de la sécrétion de $L H$ chez des brebis allaitantes en réponse d l'introduction d'un bélier 3 semaines après la mise-bas (Poindron, et al., 1979). 
Lors de l'œestrus, l'association avec le bélier réduit d'environ 1/3 la durée de la réceptivité sexuelle (Zeltobrujk et Rak, 1964 ; Parsons et Hunter, 1967 ; Van der Westhuisen, Van Niekerk et Hunter, 1970). Il en est de même chez la truie (Signoret, du Mesnil du Buisson et Mauléon, 1972). Cet effet ne nécessite pas le contact direct (Parsons et Hunter, 1967). Ce phénomène apparaît même lorsque le comportement d'cestrus est provoqué par injection hormonale à une brebis ovariectomisée (Fletcher et Lindsay, 1971). L'activité sexuelle elle-même n'esł pas en cause puisque l'effet est le même si le contact n'a lieu que pendant les 24 h suivant l'injection d'œstrogènes c'est-àdire avant que n'apparaisse la réceptivité sexuelle de la femelle. Le contact physique n'est pas nécessaire mais le canal sensoriel olfactif n'est pas seul en cause : des brebis anosmiques réagissent comme des témoins lorsqu'elles sont en présence du mâle (Signoret et Lindsay, non publié).

Les résultats passés en revue par Van der Westhuisen, Van Niekerk et Hunter (1970) font apparaître des résultats souvent opposés, en ce qui concerne l'action sur l'ovulation (Parsons, Hunter et Rayner, 1967 ; Cahill et al., 1974). La maîtrise, grâce à un traitement de progestagènes, des processus qui conduisent à l'ovulalion (Robinson, 1965 ; Cognié, Mariana, Thimonier, 1970) permet de disposer d'une situation où la chronologie des événements est connue avec une grande précision. Dans ce cas, il a été possible de montrer que la présence du bélier accélère d'environ $8 \mathrm{~h}$ le processus de l'ovulation, en modifiant d'aułanl le moment de la décharge de LH (Lindsay et al., 1975). Ce dernier phénomène apparaît également lorsque la sécrétion de LH est la conséquence d'une injection d'œstrogènes à une brebis ovariectomisée prétraitée à la progestérone (Signoret, 1974). Cette réponse ne nécessite pas le contact physique et n'implique pas nécessairement le canal sensoriel olfactif.

Les résultats semblent pouvoir être étendus à d'autres espèces : chez les bovins, l'ovulation pourrait être influencée par la présence du mâle même sans accouplement (Ayalon et Weis, 1970) ef la durée de réceptivité observée en présence permanente du taureau paraît beaucoup plus brève (moins de $3 \mathrm{~h}$ ), selon (Sambraus, 1968) que ce qui est généralement cité chez des vaches isolées (12 à 24 h).

Chez la truie, l'accouplement accélère d'environ $4 \mathrm{~h}$ et regroupe les ovulations (Signoret, du Mesnil du Buisson et Mauléon, 1972). Enfin, chez la Souris (Zarrow, Christenson et Eleftheriou, 1971 ; Zarrow, Eleftheriou et Denenberg, 1972) des stimulis olfactifs venant du mâle facilitent l'ovulation induite par PMSG.

\section{Conclusion.}

A divers stades, des stimulations produites par l'environnement social peuvent intervenir dans les régulations hormonales pour aboutir à modifier le déroulement temporel de ces phénomènes.

Le cycle œstrien, son démarrage lors de la puberté, ou après un anœstrus dû à la saison ou à l'allaitement, l'ovulation elle-même, sont donc susceptibles d'être influencés dans leur déroulement temporel par des stimulations sensorielles venant de l'environnement social. On peut imaginer que, dans la situation naturelle, leur infervention est la règle générale, le fonctionnement spontané de l'axe hypophyso-ovarien serait un mécanisme de sécurifé assurant, en l'absence des stimulations sociales naturelles, le déroulement des fonctions génitales. 
Ces divers effets peuvent avoir une valeur adaptative de grande importance. Lorsque la structure sociale est telle que le rapprochement mâle-femelle est de courte durée, il importe que les chances de fécondation y soient maximum : l'ajustement du moment de l'ovulation, d'autres actions possibles de la présence du mâle, et des stimulations de l'accouplement peuvent y contribuer. II est ainsi remarquable de constater le très haut niveau de fécondation obtenu après un seul œstrus chez l'animal sauvage en situation naturelle, résultat que l'on ne réussit à obtenir que dans les meilleures conditions en élevage.

Le groupage de l'activité sexuelle, donc des naissances, a également une valeur adaptative certaine, en réduisant au minimum la période où ont lieu les mise-bas pendant laquelle les individus sont le plus sensibles à la prédation. On constate ainsi que, par exemple, la quasi-tolalité des femelles de sangliers d'une même unité sociale s'accouplent en quelques jours, toutes les naissances ayant lieu en 4 à 5 jours (Meynhardt, 1978). De même, chez les ovins primitifs de Soay observés par Grubb (1971), les œstrus sont groupés dans chaque unité sociale d'une manière parfois indépendante. Un tel mécanisme peut résulter de l'effet de la présence des mâles, qui, normalement occupent des domaines vitaux séparés de ceux des femelles et ne les rejoignent qu'au moment du rut (Grubb et Jewell, 1966 ; Meynhardt, 1978). Ceci suppose une régulation d'une très grande précision intervenant à la fois sur l'apparition de l'ovulation des adultes et la puberté des jeunes.

Nous nous sommes limités à envisager les stimulations du mâle sur la femelle. C'est, en effet, chez elle, en raison de la précision temporelle des phénomènes du cycle œstrien et de l'ovulation, que leur effet est le plus clair. Toutefois, la possibilité d'une action inverse - effet de la femelle sur la physiologie sexuelle du mâle - et celle de l'ensemble des relations sociales sur les reproducteurs des deux sexes apparaissent de plus en plus fréquemment lorsque sont mieux connus les phénomènes de la reproduction dans des espèces sauvages variées.

L'ensemble des interrelations de l'animal avec ses congénères et son milieu — son insertion éco-éthologique - semble ainsi intervenir dans les mécanismes de la reproduction d'une manière dont la précision des connaissances acquises au laboratoire nous avait peut-être fait sous-estimer l'importance.

Présenté au Colloque D.G.R.S.T. de Port Bail, 27 février-1er mars 1979.

Accepté en novembre 1979.

\section{Références}

ALBRECHT E. D., 1967. Source of the pheromone causing estrous synchronization in the laboratory mouse. Ph. D Thes. Univ. Vermont.

ARON C., CHATEAU D., 1969. Données nouvelles sur le comportement précoce d'acceptation de la ratte au cours de cycles de 5 jours. C. R. Soc. Biol. Paris, 163, 1699-1702.

ARON C., CHATEAU D., 1971. Presumed involvement of pheromones in mating behavior in the rat. Horm. Behav., 2, 315-323.

ARON C., ROOS J., ASCH G., 1968. Données statistiques ef biologiques nouvelles sur le comportement d'acceptation de la ratte au cours du cycle œstral. Rev. Comp. anim., 2, 52-71.

AYALON N., WEIS Y., 1970. The influence of a teaser bull on estrus detection. Refuah vet., 27, $22-25$. BOURN R., KINSEY R., CARLSON R., ZIMMERMAN D. R., 1975. Puberty in gilts as influenced by boar exposure and « transport phenomenon ». J. Anim. Sci., 41, 344. 
BRADEN A. W. H., MOULE G. R., 1964. Effects of stress on ovarian morphology and cestrous cycles in ewes. Aust. J. agric. Res., 15, 937-949.

BRONSON F. H., DESJARDIN C., 1974. Circulating concentrations of FSH, LH, œestradiol and progesterone associated with acute male-induced puberty in female mice. Endocrinology, 4, 634-637.

BRONSON F. H., MARUNIAK J. A., 1975. Male induced puberty in female mice : evidence for a synergistic action of social cues. Biol. Reprod., 13, 94-98.

BRONSON F. H., WHITTEN W. K., 1968. Oestrous-accelerating pheromone of mice : assay androgen-dependency and presence in bladder urine. J. Reprod. Fertil., 15, 131-134.

BROOKS P. H., COLE D. J. A., 1970. The effect of the presence of a boar in the attainment of puberty in gilts. J. Reprod. Fertil., 23, 435-440.

CAHILL L. P., BUCKMASTeR J. M., CUMMING I. A., PARR R. A., WILLIAMS A. H., 1974. The effect of the presence of a ram on the time of ovulation in ewes. J. Reprod. Fertil., 40, 475-477.

CHESWORTH J. M., TAIT A., 1974. A note on the effect of the presence of rams upon the amount of luteinizing hormone in the blood of ewes. Anim. Prod., 19, 107-110.

CHIPMAN R. K., ALBRECHT E. D., 1974. Regulatory effect of urinary pheromones on puberty in the mouse. Biol. Reprod., 11, 268-279.

CHIPMAN R. K., FOX K. A., 1966. Oestrus synchronization and pregnancy blocking in wild house mice (Mus musculus). J. Reprod. Fert., 12, 233-236.

COGNIÉ Y., MARIANA J. C., THIMONIER J., 1970. Ełude du moment d'ovulation chez la brebis normale ou traitée par un progestagène associé ou non à une injection de PMSG. Ann. Biol. anim. Bioch. Biophys., 10, 15-24.

COGNIÉ Y., POINDRON P., ORGEUR P., 1978. Rupture de la période de repos sexuel saisonnier chez la brebis par l'effet bélier et le blocage de la sécrétion de prolactine. Journ. Rech. Ovine, Caprine, Itovic éd. Paris, 339-349.

COLBY D. R., VANDENBERGH J. G., 1974. Regulatory effects of urinary pheromones on puberty in the mouse. Biol. Reprod., 11, 268-279.

COOPER K. J., HAYNES N. B., 1967. Modification of the œstrus cycle of the underfed rat associated with the presence of the male. J. Reprod. Fertil., 14, 317-320.

DRICKAMER L. C., 1974. Contact stimulation, androgenized females and accelerated sexual maturation in female mice. Behav. Biol., 12, 101-110.

DYRMUNDSSON O. R., LEES J. L., 1972. Effect of rams on the onset breeding activity in clun forest ewe lambs. J. agric. Sci. Camb., 79, 269-271.

FERNANDEZ-BACA S., SUMAR J., NOVOA C., 1972. Comportamiento sexual de la alpaca macho frente a la renovacion de las hembras. Rev. Inv, Pec. (IVITA) Univ. Nac. S. Marcos, 1, 115-128.

FLETCHER I. C., LINDSAY D. R., 1971. Effect of rams on the duration of œstrous behavior in ewes. J. Reprod. Ferf. 25, 253-259.

GRUBB P., JEWELL P. A., 1966. Social, grouping and home range in feral soay sheep. Symp. Zool. Soc. Lond., 18, 179-210.

GRUBB P., 1971. Mating activity and the social significance of rams in a feral sheep community, 457-476. In The behaviour of ungulates and its relation to management, Univ. Calgary, Alberta (Canada).

HUGHES R. L., 1964. Effect of changing cages, introduction of the male and other procedures on the cestrous cycle in the rat. C.S.I.R.O. Wild Res., 9, 115-122.

KENNEDY J. M., BROWN K., 1970. Effects of male odor during infancy on the maturation, behavior and reproduction of female mice. Develop. Psychol., 3, 179-189.

KNIGHT T. W., PETERSON A. J., PAYNE E., 1978. The ovarian and hormonal response of the ewe to stimulation by the ram early in the breeding season. Theriogenology, 10, 343-353.

LAMOND D. R., 1959 . Effect of stimulation derived from other animals of the same species on œstrus cycles in mice. J. Endocr., 18, 343-349.

LINDSAY D. R., COGNIÉ Y., PELLETIER J., SIGNORET J. P., 1975. Influence of the presence of rams on the timing of ovulation and discharge of LH in ewes. Physiol. Behav., 15, 423-426.

LISHMAN A. W., HUNTER G. L., 1966. Sexual activity in the ewe following isolation from the ram during the breeding season. S. Afr. J. agric. Sci., 9, 993-1003.

MARSDEN H. M., BRONSON F. H., 1964. Estrous synchrony in mice : alteration by exposure to male urine. Science, 144, 1469. 
MAULÉON P., DAUZIER L., 1965. Variations de durée de l'ancestrus de lactation chez les brebis de race lle-de-France. Ann. Biol. anim. Bioch. Biophys., 5, 131-143.

MESNIL du BUISSON F., du SIGNORET J. P., 1962. Influences externes sur le déclenchemen† de la puberté chez la truie. Ann. Zootech., 11, 53-59.

MEYNHARDT H., 1978. Schwarzwild-report. Neumann Verlag Leipzig Radebeul.

OLDHAM C. M., 1980. A study of sexual and ovarian activity in merino sheep. Ph. D Thes. Univ. West. Austr.

OLDHAM C. M., MARTIN G. B., 1979. Stimulation of seasonally anovular merino ewes by rams. II. Premature regression of raim-induced corpora lufea. Anim. Reprod. Sci., 1, 291-295.

OLDHAM C. M., MARTIN G. B., KNIGHT T. W., 1979. Stimulation of seasonally anovular merino ewes by rams. I. Time from introduction of the rams to the pre-ovulatory $\mathrm{LH}$ surge and ovulation. Anim. Reprod. Sci., 1, 283-290.

PARSONS S. D., HUNTER G. L., 1967. Effect of the ram on duration of cstrus in the ewe. J. Reprod. Fertil., 14, 61-70.

PARSONS S. D., HUNTER G. L., RAYNER A. A., 1967. Use of probit analysis in a study of the effect of the ram on time of ovulation in the ewe. J. Reprod. Fertil., 14, 71-80.

PATERSON A.M., 1978. Oestrus and ovarian activity in gilts induced into early puberty by the introduction of mature boars. Proc. austr. Soc. Anim. Prod., 12, 246.

POINDRON P., COGNIÉ Y., GAYERIE F., ORGEUR P., OLDHAM C. M., RAVAULT J. P., 1980. Introducing rams to seasonnaly or lactationally anovular ewes causes a rapid modification of their secretion of LH, but not FSH, followed by ovulation. Physiol. Behav. (to be published).

PRUD'HON M., DENOY I., 1969. Effets de l'introduction de béliers vasectomisés dans un troupeau Mérinos d'Arles 15 jours avant le début de la lutte de printemps, sur l'apparition des œstrus, la fréquence des erreurs de détection des ruts et la fertilité des brebis. Ann. Zoofech., 18, 95-106.

RICHES J. M., WATSON R. M., 1954. The influence of the introduction or rams on the incidence of cstrus in Merinos ewes. Austr. J. Agric. Rdes., 5, 141-147.

ROBINSON T. J., 1965. Use of progestagen-impregnated sponges inserted intravaginally or subcutaneously for the control of the ostrus cycle in the sheep. Nature (Lond.), 206, 39-41.

ROWLINSON R., BRYANT M. J., 1974. Un système permettant de maitriser l'anœstrus de lactation Effet du mâle. In Congr. int. Med. vét, porc., Lyon, C5.

SAMBRAUS H. H., 1968. Das Sexualverhalten des Rindes bein freier Herden-haltung, pp. 1667-1670. In V/e Congr. int. Reprod. anim., Insém. artif., Paris, 2.

SHELTON M., 1960. Influence of the presence of a male goat on the initiation of cestrus cycling and ovulation of angora does. J. Anim. Sci., 19, 368-375.

SIGNORET J. P., 1970. Reproductive behaviour of pigs. J. Reprod. Fertil., suppl. 11, 105-117.

SIGNORET J. M., 1974. Influence of the presence of rams on the luteinizing hormone surge after œstradiol benzoate injection in ovariectomized ewes. J. Endocr., 64, 589-590.

SIGNORET J. P., du MESNIL du BUISSON F., MAULEON P., 1972. Effect of mating on the onset and duration of ovulation in the sow. J. Reprod. Fert., 31, 327-330.

UNDERWOOD E. J., SHIER F. L., DAVENPORT N., 1944. Studies in sheep husbandry in Western Australia. V. The breeding season of Merino, crossbred and British breed ewes in the agricultural districts. J. Dep. Agric. West. Aust., 21, 135-143.

VANDENBERGH J. G., 1967. Effect of the presence of a male on the sexual maturation of female mice. Endocrinology, 81, 345-349.

VANDENBERGH J. G., 1969. Male odor accelerates female sexual maturation in mice. Endocrinology, 84, 658-660.

VANDENBERGH J. C., DRICKAMER L. C., COLBY D. R., 1972. Social and dietary factors in the sexual maturation of female mice. J. Reprod. Fertil., 28, 397-405.

VANDENBERGH J. C., WHITSETT J. M., LOMBARDI J. R., 1975. Partial isolation of a pheromone accelerating puberty in female mice. J. Reprod. Fertil., 43, 515-523.

VAN DER LEE S., BOOT L. M., 1955. Spontaneous pseudopregnancy in mice. Acta physiol. pharmacol. néerl., 5, 213-214.

WATSON R. H., RADFORD H. M., 1960. The influence of rams on onset of cstrus in Merino-ewes in the spring. Austr. J. agric. Res., 11, 65-71. 
VAN DER WESTHUISEN J. M., VAN NIEKERK C., HUNTER G. L., 1970. Duration of cstrus and time of ovulation in sheep : effect of synchronization, season and ram. Agroanimalia, 2, $131-138$.

WHITTEN W. K., 1956. Modifications of the œstrous cycle of the mouse by external stimuli associated with the male. J. Endocr., 13, 399-404.

WHITTEN W. K., 1958. Modification of the cestrous cycle of the mouse by external stimuli associated with the male. Changes in the ostrous cycle determined by vaginal smears. J. Endocr., 17, 307-313.

WHITTEN W. K., 1959. Occurrence of ancestrus in mice caged in groups. J. Endocrinol. 18, 102-107. WHITTEN W. K., 1966. Pheromone and mammalian reproduction. Adv. Reprod. Physiol., 1, 155-177.

ZARROW X., CHRISTENSON C. M., ELEFTHERIOU B. E., 1971. Strain differences in the ovulatory response of immature mice to PMS and to the pheromonal facilitation of PMS-induced ovulation. Biol. Reprod., 4, 52-56.

ZARROW M. X., ELEFTHERIOU B. E., DENENBERG V. H., 1972. Pheromonal facilitation of HCGinduced ovulation in different strains of mice. Biol. Reprod., 6, 277-280.

ZELTOBRUJK N. A., RAK L. A., 1964. Stimulation neuro-sexuelle de la fonction reproductive des brebis (en russe). Ovisevodstvo, 10, 8-11. 